OPEN ACCESS

Edited by:

Jan Kassubek

University of UIm, Germany

Reviewed by:

Chuanming Li,

Chongqing Medical University, China

Xudong Shen,

Guizhou Medical University

(GMU), China

*Correspondence:

Amin F. Saad

asaad@americanrad.com

Specialty section:

This article was submitted to

Applied Neuroimaging,

a section of the journal

Frontiers in Neurology

Received: 02 July 2019

Accepted: 22 August 2019

Published: 04 September 2019

Citation:

Saad AF, Chaudhari R and Wintermark M (2019) Imaging of Atypical and Complicated Posterior

Reversible Encephalopathy

Syndrome. Front. Neurol. 10:964

doi: 10.3389/fneur.2019.00964

\section{Imaging of Atypical and Complicated Posterior Reversible Encephalopathy Syndrome}

\author{
Amin F. Saad ${ }^{1 *}$, Ruchir Chaudhari ${ }^{2}$ and Max Wintermark ${ }^{2}$ \\ ${ }^{1}$ Department of Radiology, Baylor University Medical Center, Dallas, TX, United States, ${ }^{2}$ Department of Radiology, Stanford \\ University, Stanford, CA, United States
}

Posterior reversible encephalopathy syndrome (PRES) is a condition clinically characterized by headache, altered mental status, seizures, and visual loss and may be associated with systemic hypertension, preeclampsia/eclampsia, chemotherapy, immunosuppressive therapies in the setting of organ transplantation, and uremic encephalopathy. While brain imaging in patients with PRES typically reveals symmetric vasogenic edema within the parietal and occipital lobes, PRES may present with atypical imaging findings such as central brainstem and deep gray involvement without subcortical edema, and even spinal cord involvement. Additionally, PRES may be complicated in some cases by the presence of cytotoxic edema and hemorrhage. This review will serve to summarize the pathophysiologic theories and controversies underlying PRES, imaging features encountered in atypical and complicated PRES, and the implications these findings may have on patient prognosis.

Keywords: PRES (posterior reversible encephalopathy syndrome), encephalopathy, hypertension, intracranial hemorrhage, pathophsiology

\section{INTRODUCTION}

Posterior reversible encephalopathy syndrome (PRES) is a syndrome affecting the CNS with a range of clinical presentations, most often including headache, altered mental status, seizures, and visual loss. PRES was first described in 1996 by Hinchey et al. (1). A multitude of conditions may lead to the development of PRES, with most common etiologies reported including moderate to severe hypertension, preeclampsia/eclampsia, infection with sepsis and shock, autoimmune disease such as systemic lupus erythematosus, multidrug chemotherapy regimens most often in the setting of hematopoietic malignancies, and in the setting of bone marrow and stem cell transplantation (2). The typical CT and MRI imaging features encountered in the setting of PRES consist of near symmetric hemispheric vasogenic edema affecting subcortical white matter and often extending to involve overlying cortex, best demonstrated with FLAIR sequences (3). Diffusion weighted imaging (DWI) usually confirms the vasogenic nature of this edema with absence of restricted diffusion. While variations exist in the most commonly encountered patterns of edema distribution, Bartynski et al. in an analysis of a large cohort of patients, described lesion distribution patterns to include a holohemispheric watershed pattern (22.8\% of 136 patients), superior frontal sulcus pattern (27.2\%), and a dominant parietal-occipital pattern (22.1\%), with partial or asymmetric expression of these primary patterns in $27.9 \%$ of patients. Notably, $98 \%$ of patients exhibited some degree of involvement of the parietal-occipital regions (4). 


\section{PATHOPHYSIOLOGY OF PRES}

The precise pathophysiologic mechanism underlying the development of PRES remains unknown, and controversy exists regarding competing mechanistic theories. The first theory describes severe hypertension which exceeds the natural autoregulatory limits of the brain $(150-160 \mathrm{~mm} \mathrm{Hg})$, with resultant injury to the capillary bed, fluid egress, and resultant vasogenic edema. This theory is supported by the common occurrence of hypertension encountered in patients with PRES (50-70\%) (5), animal studies demonstrating the development of vasogenic edema and hyperperfusion with experimentally elevated blood pressure (6), and reports of hyperperfusion in patients imaged with Tc99m-HMPAO single-photon emission CT (SPECT) (7). Problems with this theory include the development of PRES in patients with normal or only mildly increased blood pressure, studies demonstrating hypoperfusion in PRES, and a lack of correlation with the degree of brain edema and the severity of hypertension (5).

A competing theory of PRES pathophysiology describes the development of vasoconstriction due to autoregulatory compensation of severe hypertension leading to reduced brain perfusion, ischemia, and the development of vasogenic edema (8). In this theory, if left untreated or severe, the resultant ischemia may go on to frank infarction, with development of diffusion restriction. This theory is supported by the development of PRES in systemic conditions characterized by endothelial injury and a typical lack of severe hypertension such as sepsis, following bone marrow transplantation, and systemic chemotherapy. Additionally, evidence of vasculopathy in the setting of PRES as demonstrated using catheter angiography with vasoconstriction and reduced perfusion supports this theory, as does the common occurrence of PRES imaging features in a watershed distribution. Finally, imaging studies using MR perfusion have demonstrated hypoperfusion in $\operatorname{PRES}(9,10)$.

A third theory attempting to explain the development of PRES is immune system activation with a resultant cascade which induces endothelial dysfunction. In this theory, cytokines such as tumor necrosis factor alpha and interleukin-1 are released due to a systemic insult, which serve to induce expression of adhesion molecules which interact with circulating leukocytes and trigger the release of reactive oxygen species and proteases, leading to endothelial damage and fluid leakage (11). Additionally, these cytokines cause astrocytes to produce vascular endothelial growth factor (VEGF), which causes an increase in blood brain barrier permeability through the weakening of endothelial cell tight junctions, and has been shown to also activate the vesiculo-vacuolar organelle providing a major route for the extravasation of fluids and macromolecules (12). Marra et al. (11) note that increased circulating levels of VEGF in pre-eclamptic patients, a syndrome significantly associated with PRES, result in a 5-fold increase in vascular permeability (13). Increased levels of leukocyte adhesion molecules have also been associated with preeclampsia, allogenic bone marrow transplantation, solid organ transplantation, and infection/sepsis/shock (5). Brain biopsy in a case of PRES following cardiac transplantation showed endothelial activation,
T-cell trafficking, and endothelial VEGF expression (14). In this theory, hypertension and vasoconstriction are both consequences and not primary causative factors in PRES pathogenesis $(11,15)$.

A recently published theory of the pathophysiology of PRES is that of arginine vasopressin (AVP) hypersecretion (16). Multiple clinical conditions associated with the development of PRES, such as eclampsia and sepsis, are associated with AVP hypersecretion. Largeau et al. thus theorize that this increase in AVP secretion or AVP receptor density results in activation of vasopressin V1a with associated cerebral vasoconstriction, endothelial dysfunction, and cerebral ischemia with resultant cytotoxic edema. This may then lead to increased endothelial permeability and subsequent vasogenic edema (16). This theory may open the possibility for pharmacologic therapies for PRES targeting the AVP axis.

\section{ATYPICAL REGIONAL INVOLVEMENT IN PRES}

While PRES most commonly manifests on imaging as subcortical/cortical edema within the cerebral hemispheres with a parietal-occipital predominance and some variable involvement of deep structures as well as the posterior fossa, it may occur in an atypical fashion (Figure 3) with isolated involvement of deep gray nuclei, brainstem/cerebellar hemispheres, and exceptionally the spinal cord without cerebral hemispheric involvement. These findings may lead to a diagnostic dilemma, with a delay in diagnosis and reversal of the offending condition potentially leading to a poor patient outcome. In a series of 124 patients with PRES, McKinney et al. noted $4 \%$ of patients had imaging findings of a "central variant" PRES, revealing brainstem or deep gray nuclei involvement without involvement of the cerebral hemispheres (17). In an additional series by McKinney et al. (18) consisting of 76 patients, involvement included the thalamus (30.3\%), cerebellum (34.2\%), brainstem (18.4\%), and basal ganglia (11.8\%) with unilateral involvement seen in 2.6\%. Liman et al. (19) studied a cohort of 96 patients with PRES and found deep gray nuclei involvement in $\sim 25 \%$ of patients and infratentorial involvement (predominantly cerebellar and pontine) in more than $50 \%$ of patients. These authors found a parieto-occipital pattern in 53\%, superior frontal sulcus pattern in $17 \%$, holohemispheric watershed pattern in $17 \%$, and a central pattern in $14 \%$. Another cohort of 50 patients studied by Kastrup et al. (20) demonstrated basal ganglia involvement in $1.6 \%$ of patients and cerebellar involvement in $6.5 \%$. In the few reported cases of spinal cord involvement by PRES, all patients demonstrated confluent expansile central cord T2 signal elevation spanning at least four segments, with involvement of the cervicomedullary junction (21). Five of these nine patients had supratentorial involvement, while all revealed brainstem involvement.

\section{HEMORRHAGE IN PRES}

Posterior reversible encephalopathy syndrome (PRES) may be complicated by the presence of hemorrhage (Figures 1-3), on the 


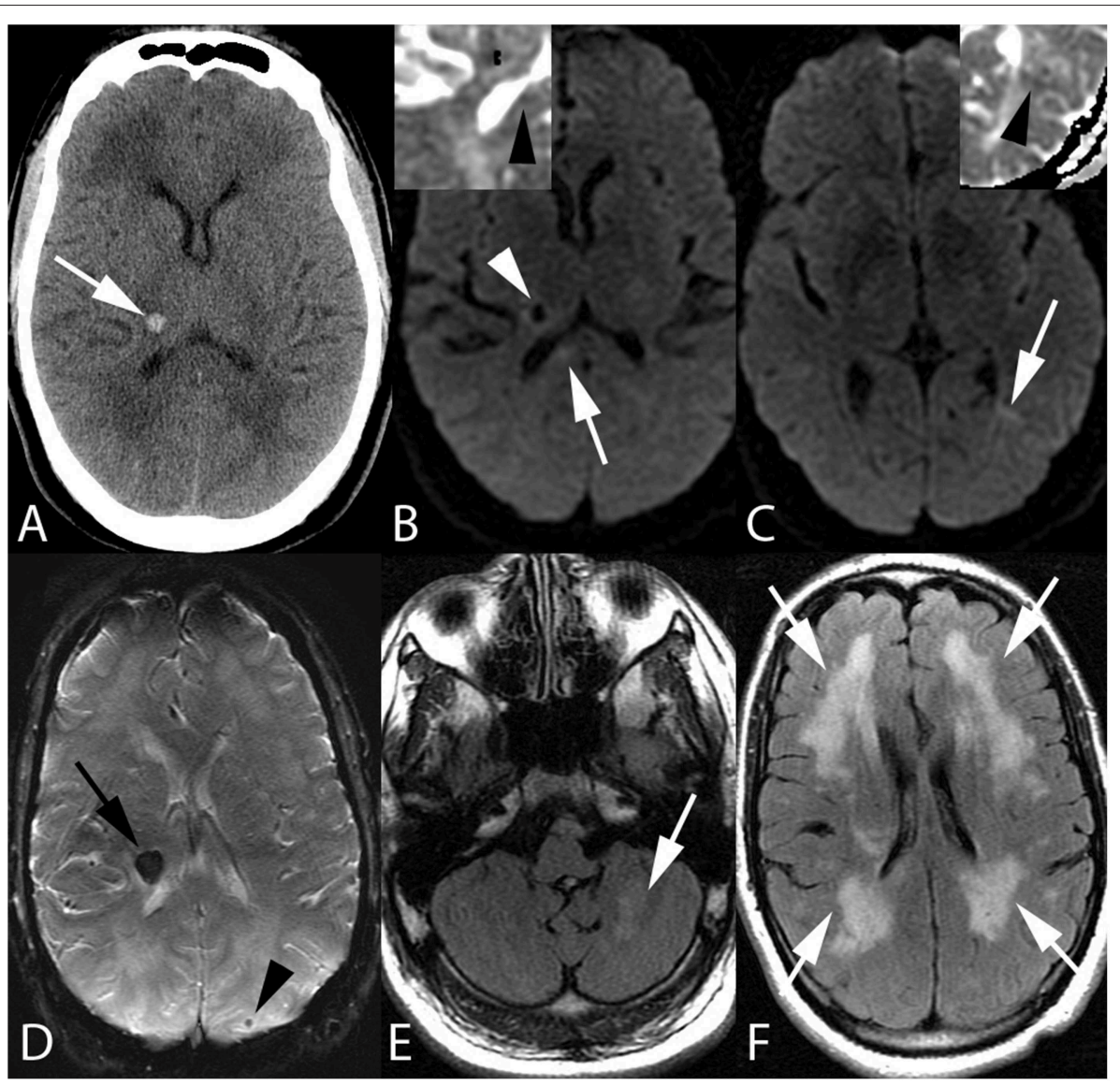

FIGURE 1 | Fifty-five-year-old man with end stage renal disease and severe hypertension. Axial CT image (A) reveals a focal parenchymal hemorrhage at the junction of the right thalamus and posterior limb of the right internal capsule (arrow). Axial DWI images with ADC inserts (B,C) show foci of diffusion restriction within the right corpus callosum splenium (arrow, B) and left temporo-occipital periventricular white matter (arrow, C). ADC maps confirm diffusion restriction (insert B,C, arrowheads). Again seen is right thalamocapsular hematoma (arrowhead, B). Axial SWI image (D) demonstrates blooming of right thalamocapsular hematoma (arrow) in addition to a punctate hemorrhage within left parietal subcortical white matter (arrowhead). Axial FLAIR images (E,F) show left cerebellar (arrow, E) and confluent bilateral frontoparietal (arrows, F) edema.

order of $15 \%$ in a series of 151 patients studies by Hefzy et al. (22) which utilized gradient echo T2* (GRE) images. In this series, focal petechial/microhemorrhages $(<5 \mathrm{~mm})$, sulcal subarachnoid hemorrhage, and focal hematoma formation were seen with equal frequency. Of note, hemorrhage was significantly more common in patients following bone marrow transplantation than in solid organ transplantation, potentially based on underlying coagulopathy, with similar increased incidence in those patients receiving systemic anticoagulation. No difference in hemorrhage incidence was seen in patients with normal, mildly elevated, or severely elevated blood pressure. In a series of 31 patients reported by McKinney et al. (23) utilizing susceptibility-weighted images (SWI), hemorrhage was more commonly detected (64.5\% of patients). Microhemorrhages were seen in $58.1 \%$ of patients at presentation and $64.7 \%$ at followup, while subarachnoid hemorrhage was seen in $12.9 \%$ and parenchymal hematoma formation was seen in $6.5 \%$. In the series reported by Liman et al. (19), microhemorrhages were seen in $14 \%$ of patients, sulcal subarachnoid hemorrhage in $4 \%$, and parenchymal hematoma formation in $11 \%$. Kastrup et al. (20) found microhemorrhages in $17 \%$ of the 29 patients who had T2* or SWI images available in their cohort. The overall rate of hemorrhage encountered in PRES range from 15 to 65\%, with the majority likely reflecting the majority of the higher reported incidences (24). The mechanism of hemorrhage in PRES may be secondary to pial vessel rupture in the setting of severe hypertension or reperfusion injury in the setting of vasoconstriction (25).

\section{DIFFUSION RESTRICTION IN PRES}

Vasogenic edema predominates in PRES, however cases may be complicated by the development of cytotoxic edema as indicated by diffusion restriction (Figure 1). Some cases may show reversibility of diffusion restriction similar to findings seen in patients with transient cerebral ischemia, venous ischemia/infarction, and vasospasm following subarachnoid hemorrhage although restriction often progresses to frank infarction with encephalomalacia identified on follow-up. In a series of 76 patients reported by McKinney et al. (18), 17.3\% 


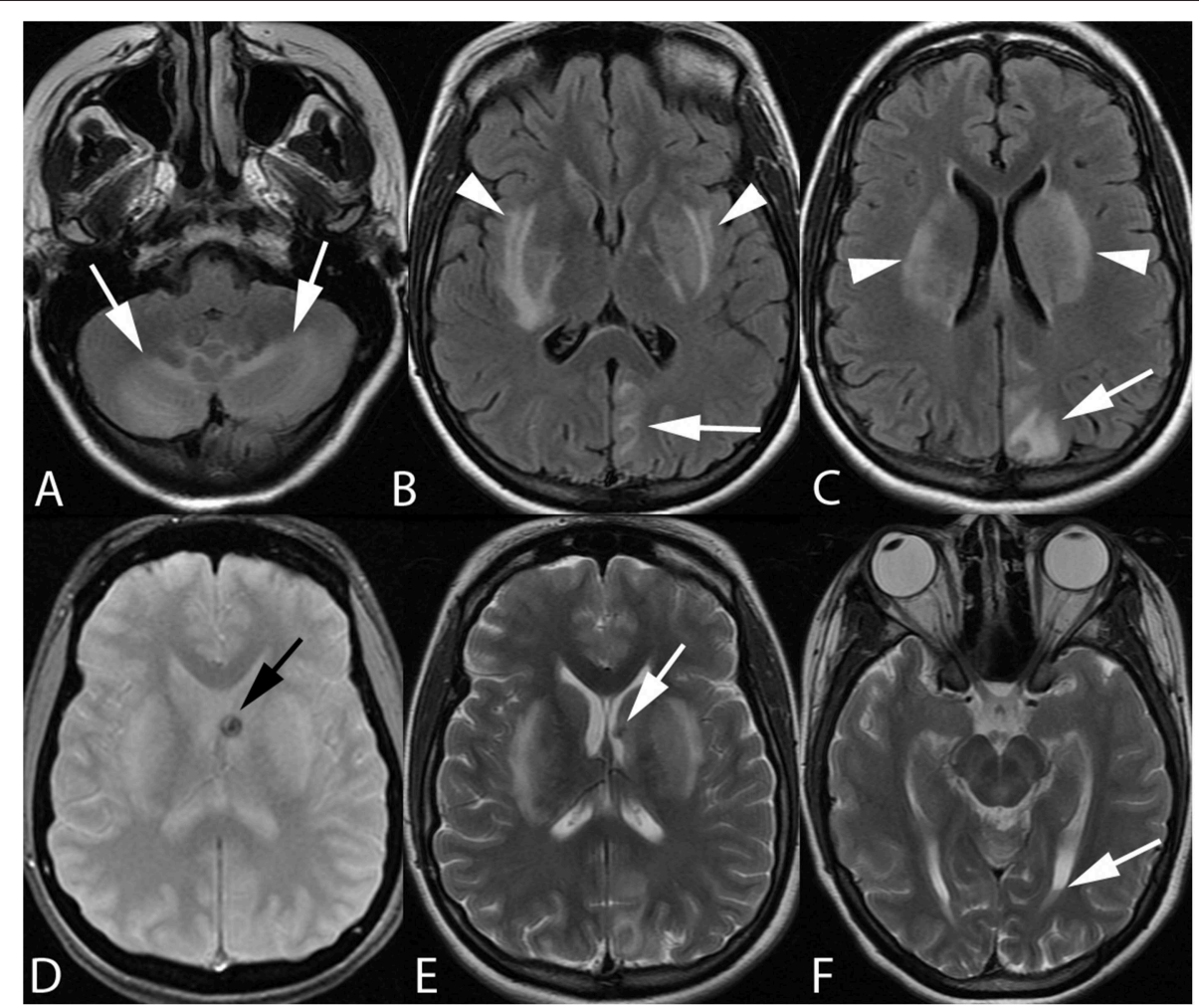

FIGURE 2 | Twenty-one-year-old pregnant woman with eclampsia. Axial FLAIR images (A-C) demonstrate bilateral cerebellar hemisphere and vermian (arrows, A), bilateral lentiform/caudate and capsular (arrowheads, B,C), and left parieto-occipital edema. Axial GRE (D) and T2-weighted (E,F) images reveal focal hemorrhage within the left caudothalamic groove (arrow, D,E) extending to the left lateral ventricular body with a small hematocrit level within the left occipital horn (arrow, F) from intraventricular extension of hemorrhage.

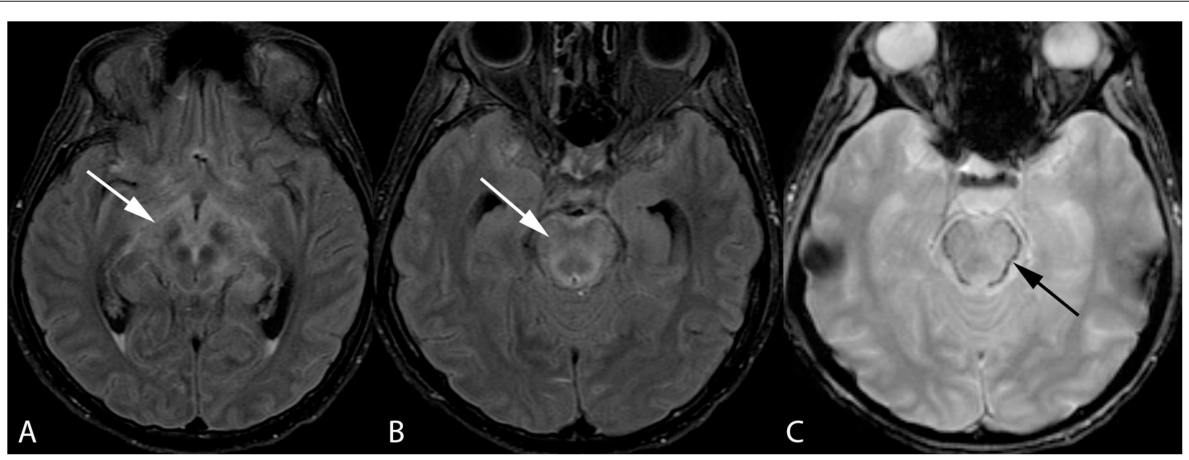

FIGURE 3 | Forty-two-year-old woman with history of bone marrow transplantation. Axial FLAIR images (A,B) demonstrate central variant PRES with edema involving the midbrain with extension to the hypothalamus and optic tracts (arrow, A) and pons (arrow, B). Axial GRE image (C) shows petechial hemorrhage at the periphery of the pons (arrow).

demonstrated areas of restricted diffusion. Covarrubias et al. (26) reported a series of 22 patients with PRES, with six patients (22\%) demonstrating abnormal diffusion signal and two patients revealing progression to infarction on follow-up. In the setting of extensive vasogenic edema encountered in PRES, some areas of cytotoxic edema amidst regions of vasogenic edema may demonstrate isointense $\mathrm{ADC}$ signal, representing $\mathrm{ADC}$ pseudonormalization (24).

\section{CONTRAST ENHANCEMENT IN PRES}

Contrast enhancement has been variably reported in the setting of PRES, typically presenting as leptomeningeal or gyral cortical enhancement (24). Enhancement was seen in $37.7 \%$ of patients studied by McKinney et al. (18), who also reported the rare occurrence of deep white matter or overlying dural enhancement. Karia et al. (27) reported enhancement in $43.7 \%$ of 135 
patients studied, with a leptomeningeal pattern in $17.8 \%$ and a leptomeningeal plus cortical pattern in $15.6 \%$. These authors found no significant association between the presence or pattern of enhancement and patient outcome of MR imaging severity of PRES.

\section{VASOSPASM IN PRES}

Vasculopathic changes are commonly encountered on vessel imaging in PRES patients. Bartynski et al. (28) found evidence of diffuse vasoconstriction, focal vasculopathy, or vessel pruning in $87 \%$ of 46 patients studied with catheter and/or MR angiography (MRA). Of 11 patients with follow up MRA examinations, seven patients revealed improvement or resolution of vasculopathic changes. It is important to note the similarity of these findings with those encountered in reversible cerebral vasoconstriction syndrome (RCVS), which shares significant clinical and radiologic features with PRES (24). Additionally, 9-38\% of patients with RCVS demonstrate reversible vasogenic edema $(29,30)$. The underlying etiologic theories of RCVS include disturbance of cerebrovascular tone and endothelial dysfunction, similar to theories of PRES pathogenesis, and the two diagnoses may reside along a spectrum of manifestations of abnormal cerebral autoregulation and/or endothelial damage (31).

\section{ESTABLISHING PATIENT PROGNOSIS IN PRES}

Although PRES is typically reversible (70-90\% of cases) (24) and patient prognosis is often positive with removal of the offending condition leading to PRES, complication by hemorrhage and/or diffusion restriction often portends a poorer patient prognosis. In the series reported by Hefzy et al. (22), 23\% of patients with PRES complicated by hemorrhage had a poor clinical outcome, with death of six of the seven patients. In the series by Covarrubias et al. (26), death was seen in $50 \%$ of the patients who exhibited diffusion signal changes. Additionally, brainstem involvement by PRES is associated with a poorer outcome, with

\section{REFERENCES}

1. Hinchey J, Chaves C, Appignani B, Breen J, Pao L, Wang A, et al. A reversible posterior leukoencephalopathy syndrome. N Engl J Med. (1996) 334:494-500. doi: 10.1056/NEJM199602223340803

2. Bartynski WS. Posterior reversible encephalopathy syndrome, part 1: fundamental imaging and clinical features. AJNR Am J Neuroradiol. (2008) 29:1036-42. doi: 10.3174/ajnr.A0928

3. Casey SO, Sampaio RC, Michel E, Truwit CL. Posterior reversible encephalopathy syndrome: utility of fluid-attenuated inversion recovery $\mathrm{MR}$ imaging in the detection of cortical and subcortical lesions. AJNR Am J Neuroradiol. (2000) 21:1199-206.

4. Bartynski WS, Boardman JF. Distinct imaging patterns and lesion distribution in posterior reversible encephalopathy syndrome. AJNR Am J Neuroradiol. (2007) 28:1320-7. doi: 10.3174/ajnr.A0549

5. Bartynski WS. Posterior reversible encephalopathy syndrome, part 2: controversies surrounding pathophysiology of vasogenic edema. two of three patients who died despite having no diffusion changes in the series by Covarrubias et al. (26) demonstrating extensive brainstem edema. In a review of PRES cases performed by Schweitzer et al. (32), 99 cases of PRES were analyzed for vasogenic edema, hemorrhage, and diffusion restriction. Areas of vasogenic edema were given discrete variables from 1 to 10 based on regional involvement, and the term "extensive vasogenic edema" was defined as involvement of five or more areas. Hemorrhage was categorized based on the presence or absence of mass effect, and diffusion restriction was confirmed with ADC maps. "Advanced radiologic PRES" was defined as at least one of the following: extensive vasogenic edema, diffusion restriction, or hemorrhage with mass effect. Patient outcomes were based on discharge disposition: home or rehabilitation vs. death or hospice, as well as modified Rankin scale (mRS) with an mRS of 3-6 considered a poor outcome. These investigators found that extensive vasogenic edema, presence of hemorrhage, and diffusion restriction (all criteria for "advanced radiologic PRES") were associated with poor clinical outcomes in terms of both hospital discharge and mRS. In this study, brainstem edema was not associated with a poor $\mathrm{mRS}$ at discharge.

\section{CONCLUSION}

Posterior reversible encephalopathy syndrome (PRES) is a condition commonly encountered in clinical practice, with prompt recognition and intervention to remove precipitating factors serving to optimize patient outcomes and reverse symptoms as well as imaging changes. The recognition of atypical imaging manifestation of PRES is important to avoid delays in diagnosis and treatment, as is identification of complicating factors which may adversely affect patient prognosis.

\section{AUTHOR CONTRIBUTIONS}

AS wrote the majority of the manuscript. RC wrote portions of the manuscript and supplied case material. MW provided guidance and feedback in the manuscript preparation and research on the topic.
AJNR Am J Neuroradiol. (2008) 29:1043-9. doi: 10.3174/ajnr. A0929

6. MacKenzie ET, Strandgaard S, Graham DI, Jones JV, Harper AM, Farrar JK. Effects of acutely induced hypertension in cats on pial arteriolar caliber, local cerebral blood flow, and the blood brain barrier. Circ Res. (1976) 39:33-41. doi: 10.1161/01.RES.39.1.33

7. Schwartz RB, Jones KM, Kalina P, Mantello MT, Garada B, Holman BL. Hypertensive encephalopathy: findings on CT, MR imaging, and SPECT imaging in 14 cases. AJR Am J Roentgenol. (1992) 159:379-83. doi: 10.2214/ajr.159.2.1632361

8. Toole JF. Lacunar syndromes and hypertensive encephalopathy. In: Toole JF, editor. Cerebrovascular Disorders. 5th ed. New York, NY: Raven (1999). p. 342-55.

9. Brubaker LM, Smith JK, Lee YZ, Lin W, Castillo M. Hemodynamic and permeability changes in posterior reversible encephalopathy syndrome measured by dynamic susceptibility perfusion-weighted MR imaging. AJNR Am J Neuroradiol. (2005) 26:825-30. 
10. Engelter ST, Petrella JR, Alberts MJ, Provenzale JM. Assessment of cerebral microcirculation in a patient with hypertensive encephalopathy using MR perfusion imaging. AJR Am J Roentgenol. (1999) 173:1491-3. doi: 10.2214/ajr.173.6.10584788

11. Marra A, Vargas M, Striano P, Del Guercio L, Buonanno P, Servillo G. Posterior reversible encephalopathy syndromeL the endothelial hypotheses. Med Hypotheses. (2014) 82:619-22. doi: 10.1016/j.mehy.2014.02.022

12. Park SJ, Pai KS, Kim JH, Shin JI. Could increased vascular endothelial growth factor induced by interleukin 17 be the cause of posterior reversible encephalopathy syndrome in systemic lupus erythematosus? J Rheumatol. (2012) 39:870-1. doi: 10.3899/jrheum.111124

13. Bills VL, Salmon AH, Harper SJ, Overton TG, Neal CR, Jeffery $\mathrm{B}$, et al. Impaired vascular permeability regulation caused by the VEGF165b splice variant in pre-eclampsia. BJOG. (2011) 118:1253-61. doi: 10.1111/j.1471-0528.2011.02925.x

14. Horbinski C, Bartynski WS, Carson Walter E, Hamilton RL, Tan HP, Cheng S. Reversible encephalopathy after cardiac transplantation: histologic evidence of endothelial activation, T cell specific trafficking, and vascular endothelial growth factor expression. AJNR Am J Neuroradiol. (2009) 30:58890. doi: 10.3174/ajnr.A1311

15. Tetsuka S, Ogawa T. Posterior reversible encephalopathy syndrome: a review with emphasis on neuroimaging characteristics. J Neurol Sci. (2019) 404:72-9. doi: 10.1016/j.jns.2019.07.018

16. Largeau B, Le Tilly O, Sautenet B, Salmon Gandonniere C, Barin-Le Guellec C, Ehrmann S. Arginine vasopressin and posterior reversible encephalopathy syndrome pathophysiology: the missing link? Mol Neurobiol. (2019). doi: 10.1007/s12035-019-1553-y. [Epub ahead of print].

17. McKinney AM, Jagadeesan BD, Truwit CL. Central-variant posterior reversible encephalopathy syndrome: brainstem or basal ganglia involvement lacking cortical or subcortical edema. AJR Am J Roentgenol. (2013) 201:631-8. doi: 10.2214/AJR.12.9677

18. McKinney AM, Short J, Truwit CL, McKinney ZJ, Kozak OS, SantaCruz KS, et al. Posterior reversible encephalopathy syndrome; incidence of atypical regions of involvement and imaging findings. AJR Am J Roentgenol. (2007) 189:904-12. doi: 10.2214/AJR.07.2024

19. Liman TG, Bohner G, Heuschmann PU, Endres M, Siebert E. The clinical and radiological spectrum of posterior reversible encephalopathy syndrome: the restrospective Berlin PRES study. J Neurol. (2012) 259:155-64. doi: 10.1007/s00415-011-6152-4

20. Kastrup O, Schlamann M, Moenninghoff C, Forsting M, Goericke S. Posterior reversible encephalopathy syndrome: the spectrum of MR imaging patterns. Clin Neuroradiol. (2015) 25:161-71. doi: 10.1007/s00062-014-0293-7

21. Ollivier M, Bertrand A, Clarencon F, Gerber S, Deltour S, Domont F, et al. Neuroimaging features in posterior reversible encephalopathy syndrome: a pictoral review. J Neurol Sci. (2017) 373:188-200. doi: 10.1016/j.jns.2016.12.007

22. Hefzy HM, Bartynski WS, Boardman JF, Lacomis D. Hemorrhage in posterior reversible encephalopathy syndrome: imaging and clinical features. AJNR Am J Neuroradiol. (2009) 30:1371-9. doi: 10.3174/ajnr.A1588
23. McKinney AM, Sarikaya B, Gustafson C, Truwit CL. Detection of microhemorrhage in posterior reversible encephalopathy syndrome using susceptibility-weighted imaging. AJNR Am J Neuroradiol. (2012) 33:896-903. doi: 10.3174/ajnr.A2886

24. Brady E, Parikh NS, Navi BB, Gupta A, Schweitzer AD. The imaging spectrum of posterior reversible encephalopathy syndrome: a pictoral review. Clin Imaging. (2018) 47:80-9. doi: 10.1016/j.clinimag.2017.08.008

25. Doss-Esper CE, Singhal AB, Smith MS, Henderson GV. Reversible posterior leukoencephalopathy, cerebral vasoconstriction, and strokes after intravenous immune globulin therapy in guillain-barre syndrome. J Neuroimaging. (2005) 15:188-92. doi: $10.1177 / 1051228404273820$

26. Covarrubias DJ, Luetmer PH, Campeau NG. Posterior reversible encephalopathy syndrome: prognostic utility of quantitative diffusion-weighted MR images. AJNR Am J Neuroradiol. (2002) 23:1038-48.

27. Karia SJ, Rykken JB, McKinney ZJ, Zhang L, McKinney AM. Utility and significance of gadolinium-based contrast enhancement in posterior reversible encephalopathy syndrome. AJNR Am J Neuroradiol. (2016) 37:41522. doi: 10.3174/ajnr.A4563

28. Bartynski WS, Boardman JF. Catheter angiography, MR angiography, and MR perfusion in posterior reversible encephalopathy syndrome. AJNR Am J Neuroradiol. (2008) 29:447-55. doi: 10.3174/ajnr.A0839

29. Ducros A, Boukobza M, Porcher R, Sarov M, Valade D, Bousser MG. The clinical and radiological spectrum of reversible cerebral vasoconstriction syndrome. A prospective series of 67 patients. Brain. (2007) 130:3091-101. doi: 10.1093/brain/awm256

30. Singhal AB, Hajj-Ali RA, Topcuoglu MA, Fok J, Bena J, Yang D, et al. Reversible cerebral vasoconstriction syndrome: analysis of 139 cases. Arch Neurol. (2011) 68:1005-12. doi: 10.1001/archneurol.2011.68

31. Miller TR, Shivashankar R, Mossa-Basha M, Gandhi D. Reversible cerebral vasoconstriction syndrome, part 1: epidemiology, pathogenesis, and clinical course. AJNR Am J Neuroradiol. (2015) 36:1392-9. doi: 10.3174/ajnr.A4214

32. Schweitzer AD, Parikh NS, Askin G, Nemade A, Lyo J, Karimi S, et al. Imaging characteristics associated with clinical outcomes in posterior reversible encephalopathy syndrome. Neuroradiology. (2017) 59:379-86. doi: 10.1007/s00234-017-1815-1

Conflict of Interest Statement: The authors declare that the research was conducted in the absence of any commercial or financial relationships that could be construed as a potential conflict of interest.

The handling Editor declared a past collaboration with one of the authors MW.

Copyright (c) 2019 Saad, Chaudhari and Wintermark. This is an open-access article distributed under the terms of the Creative Commons Attribution License (CC BY). The use, distribution or reproduction in other forums is permitted, provided the original author(s) and the copyright owner(s) are credited and that the original publication in this journal is cited, in accordance with accepted academic practice. No use, distribution or reproduction is permitted which does not comply with these terms. 